\section{The laws of attraction: chemokines, neutrophils and eosinophils in severe exacerbations of asthma}

\section{Graziella Turato, Simonetta Baraldo, Renzo Zuin, Marina Saetta}

\section{A possible new therapeutic strategy for preventing exacerbations}

A sthma is an extremely common illness which affects an estimated 300 million people of all ages and ethnic backgrounds worldwide with significant costs for healthcare systems. ${ }^{1}$ The clinical course of the disease is characterised by acute episodes of worsening of symptoms and decrease in lung function known as exacerbations. Prevention of exacerbations has been the goal of most therapeutic interventions developed so far because exacerbations seriously affect the quality of life of patients and their families. ${ }^{2}$ The clinical presentation of asthma exacerbations may be extremely heterogeneous; they can be rapid in onset or occur more gradually. The severity of asthma exacerbations is also extremely variable, ranging from mild episodes to severe attacks which result in respiratory failure and may ultimately lead to death.

Airway inflammation is a characteristic feature consistently present in patients with asthma from the early stages in children ${ }^{3}$ to elderly people. ${ }^{4}$ The inflammatory process has been extensively investigated in stable conditions, showing an increased infiltration of eosinophils, mast cells and CD4 T lymphocytes characterised by a Th2 profile, with release of cytokines such as interleukin (IL)-4, IL-5, IL-13, eotaxin and RANTES. ${ }^{5}$ Conversely, little is known about the cellular mechanisms activated during exacerbations and, in particular, in severe exacerbations.

In this issue of Thorax ( see p 475), Qiu et $a l^{6}$ investigated the inflammatory response in bronchial biopsy specimens taken from patients admitted to hospital with a severe exacerbation of asthma and who required intubation for respiratory failure. They found that, during severe exacerbations, there was an increase in eosinophils and an even greater increase in neutrophils, resulting in similar final numbers of the two granulocyte subtypes infiltrating the airways. These results confirm previous observations on sputum ${ }^{7}$ and bronchoalveolar lavage fluid in acute severe asthma. ${ }^{8}$ A unique quality of the study by Qiu et al is that, unlike the other studies, it examined bronchial biopsy specimens obtained during exacerbations. This provides direct information on the airway tissue where inflammatory responses take place and interact with smooth muscle and mucus-secreting cells, thus contributing to airway narrowing.

Tissue inflammation in severe exacerbations of asthma has been evaluated previously, but only at post mortem examination. The pathological abnormalities observed in the airways of subjects who suffered a fatal asthma attack included both eosinophil and neutrophil infiltration. ${ }^{9}$ In particular, neutrophils predominated in those patients who died suddenly after onset of the attack, while eosinophils were more frequent in fatal events with a slow onset. ${ }^{11}$ It has been proposed that these differences in the inflammatory profile may reflect the dynamics of inflammatory cell recruitment in the airways, with the neutrophil being the predominant cell in the early phase following an inflammatory stimulus and the eosinophil being the predominant cell in the late phase. ${ }^{11}$ However, it was unclear whether these observations truly reflected the cellular mechanisms underlying the severe exacerbation or were rather due to death-related events. this issue by confirming that infiltration of neutrophils and eosinophils are indeed characteristic features of severe exacerbations of asthma.

Interestingly, in a previous paper the same authors reported that both granulocyte subtypes were increased even in severe exacerbations of chronic obstructive pulmonary disease (COPD). ${ }^{12}$ Moreover, they showed that airway neutrophilia in COPD was driven by upregulation of the chemoattractants CXCL5 (ENA-78) and CXCL8 (IL-8) and of their type I receptor (CXCRI). When they tested the hypothesis that these chemoattractants may also be crucial in exacerbations of asthma, they found that only CXCL5 was significantly upregulated during exacerbations compared with stable The study by Qiu et al sheds some light on asthma. While CXCL8 binds to both CXCR1 and CXCR2, CXCL5 binds preferentially to $\mathrm{CXCR} 2^{13}$ and, indeed, the increased expression of CXCL5 was paralleled by upregulation of CXCR2 in this study. These observations suggest that activation of the CXCL5/CXCR2 axis is a crucial event in the pathogenesis of asthma exacerbations and identifies a new potential target for anti-inflammatory strategies.

One of the most intriguing findings in this study is that, at variance with COPD, in exacerbations of asthma CXCL5 and CXCR2 were correlated with the number of eosinophils infiltrating the airways rather than with neutrophils. Of interest, human eosinophils may produce CXCL5 in vitro, an effect that is particularly evident after prolonged incubation with tumour necrosis factor $\alpha(\mathrm{TNF} \alpha){ }^{14}$ Since there is evidence that TNF $\alpha$ is increased in severe asthma, ${ }^{15}{ }^{16}$ and especially in status asthmaticus, ${ }^{17}$ it can be hypothesised that even in vivo TNF $\alpha$ may induce CXCL5 upregulation by eosinophils. This in turn would drive activation of CXCR2bearing cells such as neutrophils, thus promoting their recruitment at sites of inflammation. In this context, there is promising evidence that therapeutic strategies aimed at interfering with TNF $\alpha$ signalling may be beneficial in patients with refractory asthma, ${ }^{15} 16$ especially by reducing exacerbations, ${ }^{18}$ although this requires confirmation in larger controlled studies. ${ }^{19}$

We should be careful in interpreting the findings of Qiu et al for several reasons. First, patients with an exacerbation received corticosteroids intravenously as part of the exacerbation management and most of them had been treated with inhaled or oral corticosteroids before the exacerbations. Moreover, the subjects examined were not well matched with regard to age, since patients with an exacerbation were older than those with stable asthma and were mechanically ventilated before biopsy sampling. All these factors could have potentially enhanced airway neutrophilia. ${ }^{2021}$

More importantly, this is a crosssectional rather than a longitudinal study, so the possibility that neutrophilia was already present in those subjects before occurrence of severe exacerbations cannot be ruled out. Indeed, most of the patients with an asthma exacerbation had severe disease treated with high doses of inhaled or oral corticosteroids, while patients with stable asthma had mild disease controlled only with $\beta_{2}$-agonists on an as needed basis. This difference in the degree of severity may have influenced the inflammatory cell profile in the patients with stable asthma since there 
is evidence that tissue neutrophilia is more frequent in severe asthma. ${ }^{22}$

Despite these unavoidable limitations, the report by Qiu et al is important because it promotes a better understanding of the events contributing to the development of severe exacerbations. Indeed, this study provides the framework for the cellular changes occurring in the airway tissue and the molecular mechanisms responsible for inflammatory cell recruitment. As a consequence, these observations will encourage new research into therapeutic strategies to prevent exacerbations, one of the most important aims of asthma management.

Thorax 2007:62:465-466.

doi: 10.1136/thx.2006.070656

\section{Authors' affiliations}

Graziella Turato, Simonetta Baraldo, Renzo

Zuin, Marina Saetta, Department of

Cardiothoracic and Vascular Sciences, Section of Respiratory Diseases, University of Padova, Italy

Correspondence to: Marina Saetta, Divisione di Pneumologia, Dipartimento di Scienze Cardiologiche, Toraciche e Vascolari, Universitò degli Studi di Padova, Via Giustiniani 3, 35128 Padova, Italy; marina.saetta@unipd.it

Competing interests: None.

\section{REFERENCES}

1 Masoli M, Fabian D, Holt S, et al. The global burden of asthma: executive summary of the GINA Dissemination Committee report. Allergy 2004:59:469-78.

2 Johnston NW, Sears MR. Asthma exacerbations1: Epidemiology. Thorax 2006;61:722-8

3 Barbato A, Turato G, Baraldo S, et al. Airway inflammation in childhood asthma. Am J Respir Crit Care Med 2003; 168:798-803.

4 Fabbri LM, Romagnoli M, Corbetta L, et al. Differences in airway inflammation in patients with fixed airflow obstruction due to asthma or chronic obstructive pulmonary disease. Am J Respir Crit Care Med 2003;167:418-24

5 Jeffery PK. Remodeling and inflammation of bronchi in asthma and chronic obstructive pulmonary disease. Proc Am Thorac Soc 2004;1:176-83.

6 Qiu Y, Zhu J, Bandi V, et al. Bronchial mucosal inflammation and upregulation of CXC chemoattractants and receptors in severe exacerbations of asthma. Thorax 2007:62:475-82

7 Fahy JV, Kim KW, Liu J, et al. Prominent neutrophilic inflammation in sputum from subjects with asthma exacerbation. J Allergy Clin Immunol 1995:95:843-52.

8 Lamblin C, Gosset C, Tillie-LeBlond I, et al. Bronchial neutrophilia in patients with noninfectious status asthmaticus. Am J Respir Crit Care Med 1998;157:394-402.

9 Wark PAB, Gibson PG. Asthma exacerbations - 3: Pathogenesis. Thorax 2006;61:909-15.

10 Sur S, Crotty TB, Kephart GM, et al. Sudden-onse fatal asthma. A distinct entity with few eosinophils and relatively more neutrophils in the airway submucosa? Am Rev Respir Dis 1993;148:713-9.

11 Carroll N, Carello S, Cooke C, et al. Airway structure and inflammatory cells in fatal attacks of asthma. Eur Respir J 1996:9:709-15.

12 Qiu Y, Zhu J, Bandi V, et al. Biopsy neutrophilia, neutrophil chemokine and receptor gene expression in severe exacerbations of chronic obstructive pulmonary disease. Am J Respir Crit Care Med 2003; 168:968-75.

13 Ahuja SK, Murphy PM. The CXC chemokine growth-regulated oncogene (GRO) $\alpha,(G R O) \beta$ (GRO) $\gamma$, neutrophil-activating peptide-2, and epithelial cell-derived neutrophil-activating peptide78 are potent agonists for the type $B$, but not the type $A$, human interleukin-8 receptor. J Biol Chem 1996;271:20545-50

14 Persson T, Monsef N, Andersson P, et al. Expression of the neutrophil-activating CXC chemokine EXA78/CXCL5 by human eosinophils. Clin Exp Allergy 2003:33:531-7.

15 Howarth PH, Babu KS, Arshad HS, et al. Tumour necrosis factor $\alpha$ (TNF $\alpha$ ) as a novel therapeutic target in symptomatic corticosteroid-dependent asthma. Thorax 2005;60:1012-8.

16 Berry MA, Hargadon B, Shelley $M$, et al. Evidence of a role of tumour necrosis factor $\alpha$ in refractory asthma. N Engl J Med 2006;354:697-708.

17 Tillie-LeBlond I, Pugin J, Marquette $\mathrm{CH}$, et al. Balance between proinflammatory cytokines and their inhibitors in bronchial lavage from patients with status asthmaticus. Am J Respir Crit Care Med 1999; 159:487-94.

18 Erin EM, Leaker BR, Nicholson GC, et al. The effects of a monoclonal antibody directed against tumour necrosis factor $\alpha$ in asthma. Am J Respir Crit Care Med 2006; 174:753-62.

19 Holgate ST, Polosa R. The mechanisms, diagnosis and management of severe asthma in adults. Lancet 2006;368:780-93.

20 Martin TR. Neutrophils and lung injury: getting it right. J Clin Invest 2002; 1 10:1603-5

21 Cox G. Glucocorticoid treatment inhibits apoptosis in human neutrophils. Separation of survival and activation outcomes. J Immunol 1995; 154:4719-25.

22 Wenzel SE, Szefler SJ, Leung DYM, et al. Bronchoscopic evaluation of severe asthma persistent inflammation associated with high dose glucocorticoids. Am J Respir Crit Care Med 1997; 156:737-43.

\section{Dietary supplements and asthma: another one bites the dust}

\section{Johanna Feary, John Britton}

\section{No effect of selenium supplementation on symptoms of asthma}

$\mathrm{T}$ hroughout history, clinical observation and clinical trials have identified links between nutritional deficiency and disease. For example, scurvy was described by Hippocrates over 2000 years ago, and native cultures have known its cause and cure for centuries. The first intervention study to demonstrate the successful treatment of scurvy with citrus fruits was published in 1753 by Captain James Lind in "A Treatise of the Scurvy". Moving forward to the 20th century, one of the resounding achievements in this field has been identification of the importance of folic acid supplements in the prevention of spina bifida, leading to an overall reduction in incidence in the
Western world. The possibility that nutritional factors may play a similarly important role in the aetiology of chronic respiratory disease is therefore intriguing and has recently attracted a great deal of interest.

The aetiology of asthma remains unclear, but it is widely accepted that environmental factors play a major role and, of these, diet is a potentially important contender. Evidence for this arises from the observations that the prevalence of asthma increases as societies move from a rural subsistence towards a more Western lifestyle; this is associated among other factors, with a change in dietary pattern including adoption of a more processed and "convenienceorientated" diet. The result of this dietary change is an overall increase in the intake of refined sugars, fats and additives, and a relative reduction in the intake of complex carbohydrates and micronutrients. This change is a relatively modern phenomenon, occurring in the UK since the end of wartime rationing and also resulting from increased industrialisation of the food supply chain.

There is now an extensive literature on the relationship between diet and respiratory disease. ${ }^{1-3}$ In asthma, observational studies have shown encouraging evidence of a protective effect of several nutrients on disease prevalence and symptoms, including vitamin $\mathrm{C}^{45}{ }^{5}$ vitamin $\mathrm{E},{ }^{6}$ selenium ${ }^{7}$ and magnesium. ${ }^{8}$ However, and disappointingly in view of early promise with vitamin $\mathrm{C}$, ${ }^{9}$ these findings have not generally translated into consistently positive outcomes in intervention studies. For example, in a recent randomised placebocontrolled trial of vitamin $\mathrm{C}$, magnesium or placebo in 300 patients, we found no effect of either supplement on clinical asthma control..$^{10}$ Similarly, an intervention study of vitamin $\mathrm{E}$ in adults with asthma also showed no evidence of a benefit. ${ }^{11}$ While fish oil supplements have been 